\title{
LOCALLY CONFORMALLY KÄHLER METRICS ON KATO SURFACES
}

\author{
MARCO BRUNELLA
}

\begin{abstract}
We show that every Kato surface admits a locally conformally Kähler metric.
\end{abstract}

In this article, we will prove the following theorem, which improves and completes our previous result in $[\mathrm{Bru}]$.

THEOREM 1. Every Kato surface admits a locally conformally Kähler metric.

We refer to $[\mathrm{Bel}]$ and $[\mathrm{Bru}]$, and references therein, for some background on locally conformally Kähler metrics on complex surfaces. We just recall the following fact, which will be used below: a complex surface $S$ admits a locally conformally Kähler metric if and only if its universal covering $\widetilde{S}$ admits a Kähler metric $\omega$ which transforms conformally under the action of the deck transformations $\left(g^{*}(\omega)=c_{g} \omega, c_{g} \in \mathbb{R}^{+}\right.$, for every deck transformation $\left.g\right)$. In particular, we observe the following consequence of Theorem 1.

COROLlary 2. The universal covering of a Kato surface is Kählerian.

It would be interesting to know if this last statement holds for every compact complex surface. Note, however, that there are compact complex surfaces which do not admit locally conformally Kähler metrics [Bel]; hence, a generalization of Corollary 2 to any compact complex surface should follow a different path.

The proof of Theorem 1 is based on a point of view already exploited in [Bru, Section 2], but we will not need the stability result proved in [Bru, Section 3].

Received May 10, 2010. Accepted August 10, 2010.

2010 Mathematics Subject Classification. 32J15, 32Q30.

(C) 2011 by The Editorial Board of the Nagoya Mathematical Journal 


\section{Kato surfaces}

Let us briefly recall the definition and the construction of Kato surfaces (see [Kat], [Dlo]). Let $B_{r}$ denote the (open) ball of radius $r$ in $\mathbb{C}^{2}$, and set $B=B_{1}$. Take a sequence of blowups

$$
\pi: \widehat{B} \longrightarrow B
$$

over the origin $0 \in B$, subject to the following constraint: at each step, we blow up a point belonging to the exceptional divisor created at the previous step. Take also a holomorphic (up to the boundary) embedding

$$
\sigma: \bar{B} \longrightarrow \widehat{B}
$$

which sends the origin to a point belonging to the last exceptional divisor created by $\pi$. Set

$$
W=\widehat{B} \backslash \sigma(\bar{B}) .
$$

We can glue together the two boundary components of $\partial W=(\partial \widehat{B}) \cup(\sigma(\partial B))$ using the real analytic CR-diffeomorphism

$$
\sigma \circ \pi: \partial \widehat{B} \longrightarrow \sigma(\partial B) \text {. }
$$

The result is a minimal compact complex surface $S=S_{\pi, \sigma}$, with the first Betti number equal to 1 and the second Betti number equal to the number of blowups in $\pi$. It is called a Kato surface, or a surface with a global spherical shell. Of course, such a surface cannot be Kählerian, for its first Betti number is odd.

The following remark is very simple, yet of capital importance for our construction. For every $r \in(0,1]$, the embedding $\sigma$ sends $\bar{B}_{r}$ into $\widehat{B}_{r}=$ $\pi^{-1}\left(B_{r}\right)$; indeed, the composite map $\pi \circ \sigma: \bar{B} \rightarrow B$ fixes the origin, and hence by the Schwarz lemma it maps $\bar{B}_{r}$ inside $B_{r}$ for every $r \leq 1$, which precisely means that $\sigma\left(\bar{B}_{r}\right) \subset \pi^{-1}\left(B_{r}\right)$. If we set

$$
W_{r}=\widehat{B}_{r} \backslash \sigma\left(\bar{B}_{r}\right)
$$

then we can glue, as before, the two components of $\partial W_{r}$ using $\sigma \circ \pi$. The result is the same compact complex surface $S$. Indeed, the open subsets $W_{r}, r \in(0,1]$ may be understood as different fundamental domains in the same universal covering $\widetilde{S}$ for the same action of the deck transformations. This phenomenon is also at the origin of the fact that the classification of Kato surfaces can be reduced to the classification of germs of contracting mappings of the type $\pi \circ \sigma$ [Dlo]. In fact, we may replace the balls $B_{r}$ with any domain $U \subset B$ containing zero and such that $\sigma(\bar{U}) \subset \pi^{-1}(U)$. 


\section{A Kähler metric on $\widehat{B}$}

On $\widehat{B}$, there obviously exists a Kähler form $\omega_{0}$, smooth up to the boundary. In order to prove Theorem 1 , we first modify $\omega_{0}$ on a neighborhood of $\sigma(0)$.

Consider $\sigma^{*}\left(\omega_{0}\right)$. It is a Kähler form on $B$, which admits a potential

$$
\sigma^{*}\left(\omega_{0}\right)=\operatorname{dd}^{\mathrm{c}} \varphi
$$

with $\varphi$ smooth and strictly plurisubharmonic on $B$. Up to adding an affine (hence pluriharmonic) function, we may assume that $\varphi(0)=0$ and $(\mathrm{d} \varphi)(0)=0$. The Taylor expansion of $\varphi$ at zero has the form

$$
\varphi(z)=L(z, \bar{z})+\operatorname{Re} M(z, z)+O\left(\|z\|^{3}\right)
$$

where $L$ is the Levi form at zero and $M$ is a homogeneous polynomial of degree 2 . Because $\operatorname{Re} M$ is pluriharmonic, we may replace $\varphi$ with $\varphi-\operatorname{Re} M$, and we obtain in this way a new potential for $\sigma^{*}\left(\omega_{0}\right)$, still denoted by $\varphi$, whose Taylor expansion at zero is $L(z, \bar{z})+O\left(\|z\|^{3}\right)$. This means that $\varphi$ has a minimum point at zero, of Morse type.

We can choose $\lambda>0$ and $0<r<r^{\prime}<1$ such that the function

$$
\rho(z)=\lambda\left(1+\|z\|^{2}\right)
$$

is greater than $\varphi$ on $\bar{B}_{r}$ and smaller than $\varphi$ on $\partial B_{r^{\prime}}$. The function $\widetilde{\varphi}$ on $B$ defined by $\widetilde{\varphi}=\varphi$ on $B \backslash B_{r^{\prime}}$ and $\widetilde{\varphi}=$ regularized maximum between $\varphi$ and $\rho$ on $B_{r^{\prime}}$ is therefore smooth, strictly plurisubharmonic, and equal to $\rho$ on some neighborhood of $\bar{B}_{r}$.

The Kähler form $\sigma_{*}\left(\operatorname{dd}^{\mathrm{c}} \widetilde{\varphi}\right)$ on $\sigma(B)$ glues to $\omega_{0}$ outside $\sigma(B)$, giving a new Kähler form $\omega_{1}$ on $\widehat{B}$. By construction, this Kähler form has the property

$$
\sigma^{*}\left(\omega_{1}\right)=\mathrm{dd}^{\mathrm{c}} \rho \quad \text { on } \bar{B}_{r}
$$

\section{Another Kähler metric on $\widehat{B}_{r}$}

We now modify the previous $\omega_{1}$ on a neighborhood of $\partial \widehat{B}_{r}$. Choose $s<r$ sufficiently close to $r$ so that $\widehat{B}_{s}$ still contains $\sigma\left(\bar{B}_{r}\right)$; that is, $(\pi \circ \sigma)\left(\bar{B}_{r}\right) \subset$ $B_{s}$ : 


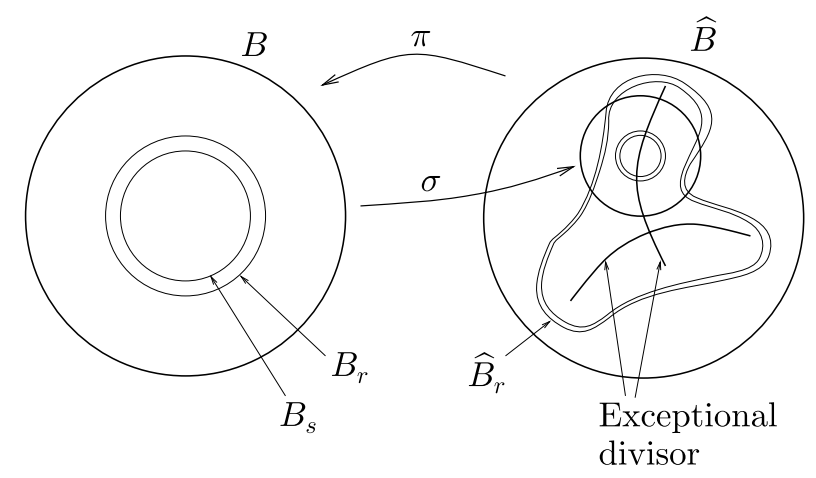

Take a potential $\psi$ for the direct image $\pi_{*}\left(\omega_{1}\right)$ :

$$
\pi_{*}\left(\omega_{1}\right)=\mathrm{dd}^{\mathrm{c}} \psi \quad \text { on } B .
$$

Note that $\psi$ is necessarily singular at 0 , but it does not matter. We can choose $c_{1} \in \mathbb{R}^{+}$and $c_{2} \in \mathbb{R}$ such that the function $c_{1} \rho+c_{2}$ is greater than $\psi$ on $\partial B_{r}$ and smaller than $\psi$ on $\partial B_{s}$. The function $\widetilde{\psi}$ on $\bar{B}_{r}$ defined by $\widetilde{\psi}=\psi$ on $B_{s}$ and $\widetilde{\psi}=$ regularized maximum between $\psi$ and $c_{1} \rho+c_{2}$ on $\bar{B}_{r} \backslash B_{s}$ is therefore smooth, strictly plurisubharmonic, equal to $c_{1} \rho+c_{2}$ on some neighborhood of $\partial B_{\widetilde{r}}$, and equal to $\psi$ on some neighborhood of $\bar{B}_{s}$.

By pulling back $\widetilde{\psi}$ with $\pi$ and taking $\mathrm{dd}^{\mathrm{c}}$, we get on $\widehat{B}_{r}$ a new Kähler form $\omega_{2}$ with the following two properties:

(1) $\omega_{2}=\omega_{1}$ on $\widehat{B}_{s} \supset \sigma\left(\bar{B}_{r}\right)$, so that $\sigma^{*}\left(\omega_{2}\right)=\operatorname{dd}^{\mathrm{c}} \rho$ on $\bar{B}_{r}$;

(2) $\pi_{*}\left(\omega_{2}\right)=\operatorname{dd}^{\mathrm{c}}\left(c_{1} \rho+c_{2}\right)=c_{1} \operatorname{dd}^{\mathrm{c}} \rho$ on some neighborhood of $\partial B_{r}$.

\section{Conformal gluing}

We can now complete the proof of Theorem 1. The gluing map $\sigma \circ \pi$ : $\widehat{B}_{r} \rightarrow \sigma\left(B_{r}\right)$ satisfies the conformal property

$$
(\sigma \circ \pi)^{*}\left(\left.\omega_{2}\right|_{U}\right)=\left.\frac{1}{c_{1}} \omega_{2}\right|_{V},
$$

where $U \subset \sigma\left(B_{r}\right)$ and $V \subset \widehat{B}_{r}$ are suitable neighborhoods of $\sigma\left(\partial B_{r}\right)$ and $\partial \widehat{B}_{r}$. Because the Kato surface $S$ is obtained by identifying these two neighborhoods via $\sigma \circ \pi$, we get on $S$ a locally conformally Kähler metric.

REMARK 3. Because $c_{1}$ can be chosen arbitrarily large, we see also that, in the terminology of [Bru, Remark 9], the open subset $I(S) \subset(0,1)$ contains an interval $(0, \varepsilon)$ (with $\varepsilon$, in principle, depending on $S$ ). We do not know if $I(S)$ is an interval. 
REMARK 4. The previous construction can be done parametrically: if $\left\{S_{t}\right\}_{t \in \mathbb{D}}$ is a family of Kato surfaces, then, up to restricting the base $\mathbb{D}$, we can find a family of locally conformally Kähler metrics $\left\{\omega_{t}\right\}_{t \in \mathbb{D}}$, smoothly depending on $t$. This is, however, slightly weaker than the stability result of [Bru], which states that every locally conformally Kähler metric $\omega_{0}$ on $S_{0}$ extends to a family $\left\{\omega_{t}\right\}_{t \in \mathbb{D}}$.

\section{REFERENCES}

[Bel] F. A. Belgun, On the metric structure of non-Kähler complex surfaces, Math. Ann. 317 (2000), 1-40.

[Bru] M. Brunella, Locally conformally Kähler metrics on certain non-Kählerian surfaces, Math. Ann. 346 (2010), 629-639.

[Dlo] G. Dloussky, Structure des surfaces de Kato, Mém. Soc. Math. France (N.S.), no. $14(1984)$.

[Kat] M. Kato, "Compact complex manifolds containing 'global' spherical shells" in Proceedings of the International Symposium on Algebraic Geometry (Kyoto, 1977), Kinokuniya, Tokyo, 1978, 45-84.

Institut de Mathématiques de Bourgogne - UMR 5584

21078 Dijon

France

brunella@u-bourgogne.fr 